\title{
Symptoms and signs of ocular surface disease related to topical medication in patients with glaucoma
}

This article was published in the following Dove Press journal:

Clinical Ophthalmology

8 April 2015

Number of times this article has been viewed

\author{
Barbara Cvenkel' \\ Špela Štunf' \\ Irena Srebotnik Kirbiš ${ }^{2}$ \\ Margareta Strojan Fležar ${ }^{2}$ \\ 'Department of Ophthalmology, \\ University Medical Center Ljubljana, \\ Ljubljana, Slovenia; ${ }^{2}$ Institute \\ of Pathology, Faculty of Medicine, \\ University of Ljubljana, Ljubljana, \\ Slovenia
}

Purpose: The aim of this study was to assess signs and symptoms of ocular surface disease (OSD) and the cytomorphological changes of ocular surface in glaucoma patients using preserved antiglaucoma drops.

Methods: In this cross-sectional study, 109 participants ( 79 patients with topical medication and 30 untreated controls) completed the Ocular Surface Diseases Index (OSDI) questionnaire and underwent an ophthalmic examination, including Schirmer test, tear film breakup time (TBUT), and fluorescein staining. Conjunctival specimens were collected by impression cytology and analyzed by light microscopy using Nelson's grading scheme (grades 0-3). This classification is based on the nucleus-to-cytoplasm ratios of epithelial cells and the numbers of goblet cells, with grade 2 considered abnormal.

Results: The medication group had significantly shorter TBUT (median [interquartile range]: 6.0 seconds [5.0-8.0 seconds] vs 9.5 seconds [6.0-12.3 seconds]; $P<0.03$ ), greater fluorescein staining $(1.0[0.75-1.25]$ vs $0[0-0.25] ; P<0.001)$, and higher impression cytology grade than the control group (median [range]: 1.0 [1:2 to $1: 6]$ vs 0.6 [1:2 to $1: 4] ; P<0.001$ ). The increasing number of drops instilled per day was associated with an increase in fluorescein staining (Spearman's rho $r=0.475 ; P<0.001)$ and shorter TBUT $(r=-0.278 ; P=0.014)$. The OSDI did not discriminate between the two groups.

Conclusion: Clinical tests and impression cytology showed ocular surface damage in patients using preserved antiglaucoma medications. However, there was no statistically and clinically significant difference in symptoms measured by OSDI score between the medication and control groups.

Keywords: ocular surface disease, dry eye diagnostic tests, ocular surface disease index questionnaire, impression cytology, topical glaucoma medication

\section{Introduction}

The purpose of glaucoma treatment is to maintain the patient's visual function and related quality of life. The quality of life is decreased in patients with bilateral advanced glaucoma but can be considerably affected by the side effects of treatment. Patients with glaucoma on long-term topical treatment have higher prevalence of signs and symptoms of dry eye..$^{1,2}$

Topical treatment is associated with side effects caused not only by the active substances but also by the preservatives and excipients. ${ }^{3,4}$ Benzalkonium chloride (BAK), the most commonly used preservative, has cytotoxic and proinflammatory effects on the ocular surface and induces squamous metaplasia of conjunctival epithelium, as well as a decrease in the number of goblet cells. ${ }^{5-8}$ Also, the recently introduced preservative polyquaternium-1 (PQ) has been shown to increase cell death and the level of proinflammatory cytokines in human corneal epithelial cell culture. ${ }^{9}$
Correspondence: Barbara Crenke Department of Ophthalmology, University Medical Center Ljubljana, Grablovičeva 46, 1000 Ljubljana, Slovenia Tel +386 I $522 \quad$ I727

Fax +386 I 5221960

Email barbara.cvenkel@gmail.com 
Clinical studies have shown that patients receiving greater number of preserved eye drops for a longer time had more severe signs and symptoms of ocular surface disease (OSD). ${ }^{1,10-12}$ Exposure to more than three drops daily of BAKpreserved medication was an independent predictor of higher Ocular Surface Disease Index (OSDI) score. ${ }^{11}$

Glaucoma patients have different tolerance to topical medication and in those with coexistent dry eye, instillation of preserved drops may deteriorate signs and symptoms of OSD and further decrease the quality of life. Additionally, side effects of treatment and intolerance to drops may reduce adherence to the prescribed treatment regimen and contribute to the progression of disease.

The aim of our study was to evaluate clinical signs of OSD using Schirmer test, tear film breakup time (TBUT), fluorescein staining, and cytological changes of conjunctival epithelial cells, as well as by subjective perception of symptoms using OSDI in glaucoma patients with topical treatment.

\section{Methods}

\section{Study design and participants}

This was a cross-sectional, case-comparison study that included glaucoma patients treated with preserved topical glaucoma medications and control subjects without any topical medications.

Glaucoma patients were recruited from a tertiary referral glaucoma clinic at the Department of Ophthalmology of the University Medical Centre, Ljubljana, Slovenia. Inclusion criteria were patients who had been taking one or more topical antiglaucoma drops for at least 6 months. Exclusion criteria were as follows: presence of active ocular inflammation or allergy, eyelid changes, ocular trauma, symptoms and any treatment of prior dry eye (ie, punctal plugs, topical corticosteroids), previous glaucoma surgery and any refractive surgery $<12$ months prior to enrollment, contact lens wearers, systemic diseases, and systemic medications inducing dry eye.

The control group included patients with ocular hypertension and relatives of glaucoma patients who were not receiving topical antiglaucoma medications, with the same before-mentioned exclusion criteria. The study adhered to the tenets of the Declaration of Helsinki and was approved by the National Medical Ethics Committee.

\section{Procedure}

Patients who met the inclusion/exclusion criteria completed the OSDI questionnaire. Clinical examination included dry eye diagnostic tests: TBUT, evaluation of conjunctival and corneal fluorescein staining, Schirmer-1 test, followed by impression cytology.

\section{OSDI questionnaire}

The OSDI is a 12-item, disease-specific quality-of-life questionnaire that is used to quantify the impact of dry eye on vision-related quality of life. It was reported to have good test-retest reliability and excellent validity, in addition to effectively discriminating among different stages of dry eye severity. ${ }^{13}$ The questionnaire includes three subscales: ocular discomfort (five items), ocular symptoms during daily activities (four items), and environmental triggers (three items). Patients are asked to recall the frequency of ocular symptoms in the previous week. The response to each item is scored from 0 , indicating "none of the time," to 4 , indicating "all of the time." Using individual item responses, the average score was transformed to a scale ranging from 0 to 100 , with higher scores representing greater disabilities.

\section{Dry eye diagnostic tests}

TBUT was measured after instillation of fluorescein with a moistened fluorescein strip (Haag-Streit AG, Köniz, Switzerland) with nonpreserved saline. The interval between the last blink and the appearance of dry spot was measured two times in succession, and the average of the two values was recorded.

The Oxford grading scheme was used to evaluate fluorescein staining of the ocular surface. Staining of ocular surface at slit lamp was compared to a series of panels with staining ranging from grade 0 (absent staining) to grade 5 (severe staining). ${ }^{14}$

Schirmer-1 test was performed without anesthetics. Schirmer strips were inserted in the inferior fornix, and the patient closed their eyes. The results were read after 5 minutes.

\section{Impression cytology of the ocular surface}

We used a $25 \mathrm{~mm}$ diameter nitrocellulose membrane filter (Filter type $0.22 \mu \mathrm{m}$; GSWP, Merck Millipore, Billerica, MA, USA) cut in half and trimmed into strips of approximately $4 \times 6 \mathrm{~mm}$. After instillation of one to two drops of topical anesthetic (Alcaine; Alcon, Puurs, Belgium) and wiping away excessive tear fluid, the strip of filter paper was gently pressed on the conjunctiva with a glass rod. After 5-10 seconds, the filter paper was peeled off and the cells were transferred by imprinting onto poly-L-lysine-coated glass slides. Specimens were collected from the inferior and temporal bulbar 
conjunctiva of the selected study eye (right eye of controls and glaucoma-treated patients if both eyes were treated; if a patient administered medication to only one eye, that eye was considered the study eye). The slides were air-dried and stained with Giemsa stain. Specimens were analyzed by light microscopy using modified Nelson's grading scheme (grades 0-3) based on the appearance of epithelial cells and the density of goblet cells. ${ }^{15}$ Grades 0 and 1 are present in normal conjunctiva with nucleus-to-cytoplasm (n/c) ratio up to $1: 3$, whereas grades 2 and 3 are abnormal $(\mathrm{n} / \mathrm{c}$ ratio greater than 1:4) and indicate squamous metaplastic changes seen in many inflammatory conditions (dry eye, use of antiglaucoma therapy, contact lens wearers). Grading was performed in a masked fashion, ie, the person (BC) performing analysis was unaware of the treatment status.

\section{Data analysis}

Statistical analyses were conducted using Statistical Package for Social Sciences (SPSS 2011, version 20, SPSS Inc, Chicago, IL, USA). Demographic variables, OSDI score, Schirmer test, TBUT, fluorescein staining grade, and impression cytology grade were compared between the control and glaucoma-treated groups. Normality of distribution for dependent variables was tested with the Shapiro-Wilk test. For normally distributed data, Student's $t$-test for independent samples was used. For nonparametric data, the MannWhitney $U$-test and the Kruskal-Wallis test were applied. Analysis of covariance with age as covariate was used to determine whether there were any statistical differences between the age-adjusted groups. The association between the number of eye drops per day and individual variables was described by the correlation coefficient (Spearman's rho). Fisher's exact test was used to test for the association between categorical variables (treatment regimen, fluorescein staining grade, and impression cytology grade). All the statistical tests were two-sided, and a $P$-value $<0.05$ was considered to be statistically significant.

\section{Results}

All glaucoma-treated patients used preserved eye drops. Among the 68 patients, the preservative was BAK only in 58 patients, BAK and PQ in six patients, or BAK and benzododecinium bromide in four patients. Eleven patients instilled topical medication preserved with PQ only (data not shown in Table 1).

Patients with antiglaucoma eye drops had shorter TBUT, an increase in fluorescein staining, and increased impression cytology grade versus the control group. The OSDI score was not different between the two groups (Table 1).

The number of eye drops per day significantly correlated with the fluorescein staining grade (Spearman's rho $=0.475$; $P<0.001$ ) and the TBUT (Spearman's rho $=-0.278 ; P=0.014$ ) (Table 2).

Increasing number of eye drops instilled per day in the group of patients with topical therapy was associated with higher grade of fluorescein staining $(P<0.001)$ and a trend of shorter TBUT $(P=0.08)$ (Figure 1A and $\mathrm{B}$ ).

Impression cytology grade was not statistically significantly different among patients taking one, two, or three and more eye drops per day $(P=0.144)$ (Figure 1C).

\section{Discussion}

Our study showed that ocular surface damage in glaucoma patients is associated with preserved topical medications. There were statistically significant differences between the glaucoma-treated patients and control group in the

Table I Characteristics of glaucoma patients taking eye drops and control subjects without drops

\begin{tabular}{|c|c|c|c|c|}
\hline & $\begin{array}{l}\text { Glaucoma group } \\
\text { with eye drops } \\
(n=79)\end{array}$ & $\begin{array}{l}\text { Control group } \\
\text { without eye } \\
\text { drops }(n=30)\end{array}$ & $P$-value & $\begin{array}{l}P \text {-value } \\
\text { adjusted } \\
\text { for age }\end{array}$ \\
\hline Mean age $\pm S D$ (years) & $70.0 \pm 11.7$ & $62.4 \pm 11.9$ & $0.003^{a}$ & \\
\hline Female-to-male ratio & $45: 34$ & $17: 13$ & $0.98^{\mathrm{b}}$ & \\
\hline \multicolumn{5}{|l|}{ Antiglaucoma medications } \\
\hline Median number of drops (quartile range), $n$ & $2(1-3)$ & - & - & \\
\hline Median duration (quartile range), months & $60(36-120)$ & - & - & \\
\hline Median of OSDI (quartile range), score & II.I (5.0-22.5) & $9.2(4.8-17.5)$ & 0.41 & 0.18 \\
\hline Median of Schirmer test (quartile range), $\mathrm{mm}$ & $10.0(5.0-24.0)$ & $10.0(5.0-7.8)$ & 0.93 & 0.19 \\
\hline Median of TBUT (quartile range), seconds & $6.0(5.0-8.0)$ & $9.5(6.0-12.3)$ & $<0.001$ & 0.03 \\
\hline Median of fluorescein staining (quartile range), grade & $1.0(0.75-1.25)$ & $0(0-0.25)$ & $<0.001$ & $<0.001$ \\
\hline Median of impression cytology (range), grade & $1.0(1: 2$ to $1: 6)$ & $0.6(1: 2$ to $1: 4)$ & $<0.001$ & $<0.001$ \\
\hline
\end{tabular}

Notes: Mann-Whitney $U$-test; analysis of covariance for age adjustment; a Student's $t$-test for independent samples; ${ }^{b}$ chi-square test; result significant at $P<0.05$. Abbreviations: OSDI, ocular surface disease index; SD, standard deviation; TBUT, tear film breakup time. 
Table 2 Correlation between treatment variables (number of eye drops per day, duration of treatment) and OSDI score, as well as signs and symptoms of dry eye, in the glaucoma-treated group

\begin{tabular}{lllll}
\hline & \multicolumn{2}{l}{ Number of eye drops per day } & & \multicolumn{2}{c}{ Duration of treatment } \\
\cline { 2 - 4 } & $\boldsymbol{r}$ & $\boldsymbol{P}$-value & $\boldsymbol{r}$ & \multicolumn{1}{c}{$\boldsymbol{P}$-value } \\
\hline OSDI, score & 0.058 & 0.61 & -0.165 & 0.147 \\
Schirmer test, mm & -0.114 & 0.32 & -0.217 & 0.055 \\
TBUT, seconds & -0.278 & 0.014 & -0.143 & 0.208 \\
Fluorescein staining, grade & 0.475 & $<0.001$ & 0.095 & 0.406 \\
Impression cytology, grade & 0.164 & 0.149 & 0.150 & 0.187 \\
\hline
\end{tabular}

Note: $P$ significant at $P<0.05$.

Abbreviations: OSDI, ocular surface disease index; TBUT, tear film breakup time; $r$, Spearman's rho correlation coefficient.

clinical tests used to diagnose OSD. In this study, TBUT was significantly reduced in glaucoma patients with topical medications as compared to that in controls. Also, glaucoma patients on treatment had higher fluorescein staining and impression cytology grades than controls without topical

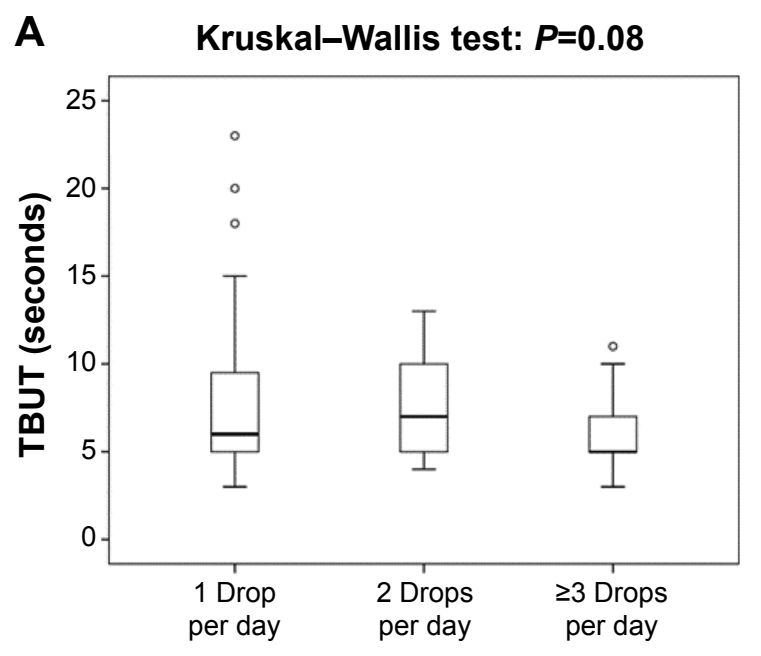

medications. However, the Schirmer test and the OSDI score were not significantly different between glaucoma-treated patients and untreated controls.

In clinical practice, the diagnosis of dry eye is commonly based on the presence of symptoms of ocular
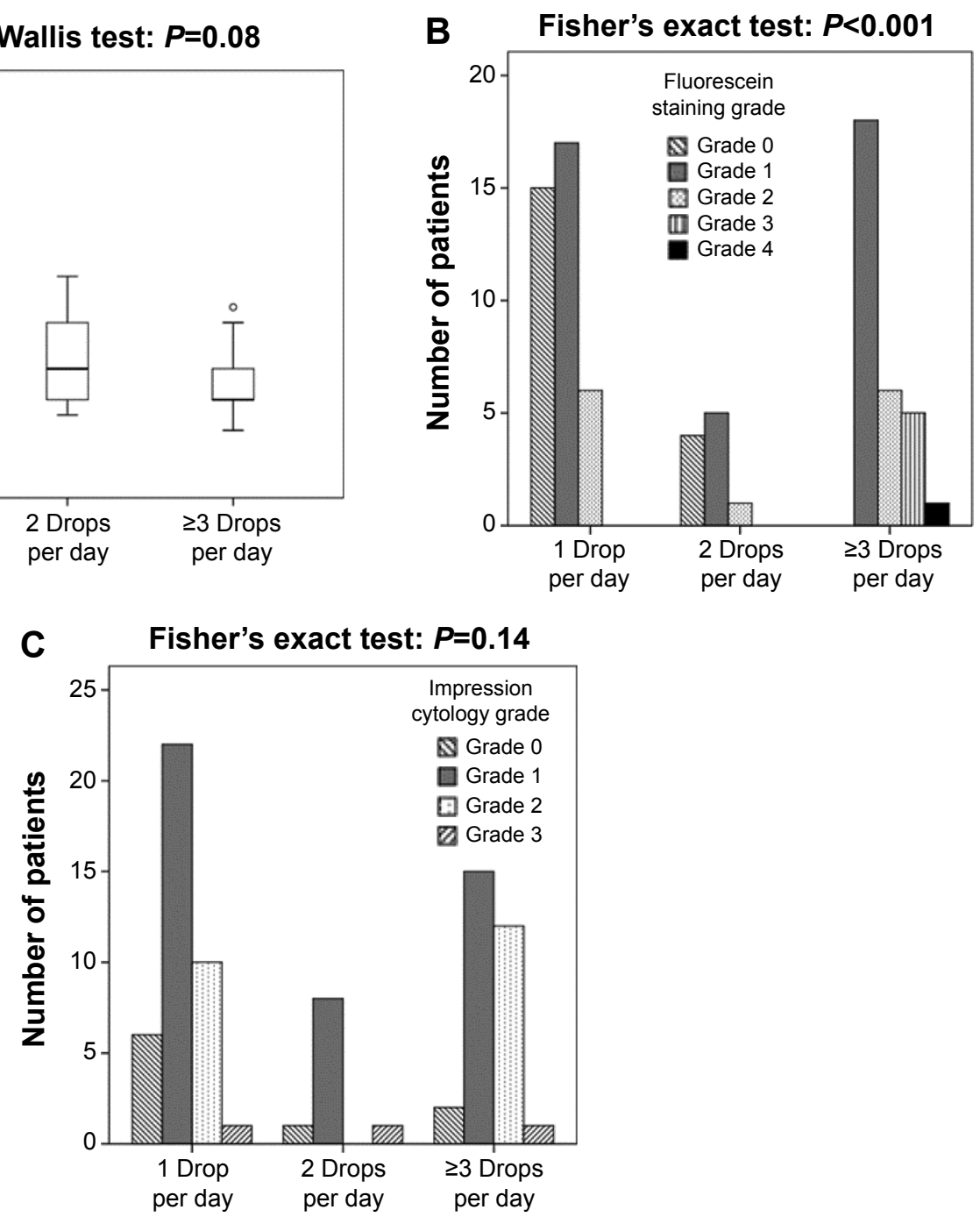

Figure I (A) Tear film breakup time (TBUT), (B) fluorescein staining, and (C) impression cytology grade among glaucoma patients with one ( $\mathrm{n}=39$ patients), two ( $\mathrm{n}=10$ patients), and three or more ( $n=30$ patients) eye drops instilled per day.

Notes: The boundaries of each box indicate the first and third quartile, the bars 1.5 times the interquartile range, and the line within each box indicates the median. Open circles indicate outliers. 
irritation, Schirmer test, TBUT, and ocular surface staining by fluorescein. In glaucoma patients, tear dysfunction is mainly attributed to the chronic administration of preserved glaucoma medications. BAK is known to damage the ocular surface, reduce the density of epithelial and goblet cells, and alter the lipid layer. These changes result in an impaired tear film with excessive evaporation. There are different findings in symptoms and signs of dry eye between glaucoma-treated patients and controls in various studies. Similar to our results, Van Went et $\mathrm{al}^{16}$ also found that only TBUT and fluorescein staining grade were significantly altered in treated patients as compared to untreated control group, but there was no significant difference for Schirmer test and OSDI. Some studies ${ }^{1,6,17,18}$ reported significantly shorter TBUT and Schirmer test, whereas others ${ }^{12,19}$ did not find any significant changes in these two clinical tests in patients treated with BAK-preserved medications. A number of factors influence the reproducibility of these tests. These factors include natural fluctuations during the day, different populations, variations in measurement techniques and scoring, systemic medications, diseases affecting ocular surface, and environmental differences. Nichols et $\mathrm{al}^{20}$ evaluated the repeatability of dry eye diagnostic tests and found that the TBUT was more repeatable than the Schirmer test and improved when the two TBUT readings were averaged.

Corneal staining with fluorescein is widely used to assess the health of the ocular surface. Our results indicate that increasing number of predominantly BAK-preserved eye drops per day was associated with increased Oxford staining score, indicating chronic cell injury. Similar findings were reported by others, that more intensive BAK-preserved topical treatment caused superficial punctate keratitis, which was present in $50 \%$ of patients treated with three drops per day. ${ }^{1,2,12,21}$ Leung et $\mathrm{al}^{2}$ found that each additional BAK-containing eye drop per day was associated with an approximately two times higher odds of showing abnormal results on the lissamine green staining test. BAK, in a dose-dependent way, reduces cell proliferation and viability and decreases corneal epithelial tight junctions. ${ }^{22,23}$ The discontinuation of preserved medication significantly reversed signs and symptoms of dry eye and reduced the frequency of superficial punctate keratitis. ${ }^{24-26}$

We found higher impression cytology grade in patients using antiglaucoma medications as compared to untreated controls. No significant difference was found among the groups with one, two, or three and more eye drops per day and the duration of treatment (Figure 1C). Most of the impression cytology studies compared small groups of patients using different types of preserved eye drops versus controls and found a decrease in goblet cell density and an increase in impression cytology grades. However, the differences among the groups of patients using different eye drops for different lengths of time were not significant. ${ }^{6,8}$ Squamous cell metaplasia is a nonspecific response of conjunctiva to a variety of toxic inflammatory stimuli. In our study, the impression cytology grades did not differ between patients using BAK/BAK combined with $\mathrm{PQ}$ or benzododecinium bromide-preserved ( 68 patients) and those using PQ-preserved (11 patients) eye drops (Mann-Whitney $U$-test; $P=0.091)$. There is a lack of data about conjunctival changes following preservative-free and non-BAK-preserved glaucoma medication. Mastropasqua et $\mathrm{al}^{27}$ evaluated only the goblet cell density in treatment-naïve eyes by impression cytology and laser scanning confocal microscopy and found an increase in conjunctival goblet cell density after preservative-free tafluprost treatment, versus patients using preserved latanoprost, and controls to the vehicle of latanoprost. Recently, a study ${ }^{28}$ compared ocular surface changes in newly diagnosed glaucoma patients using PQ-preserved or BAK-preserved travoprost. Both groups showed statistically significant increases in impression cytology grades at 1- and 6-month follow-up compared to baseline (no treatment) measurements. Interestingly, impression cytology grades were significantly higher for patients using PQ-preserved travoprost compared to the grades for patients using BAKpreserved travoprost.

Despite increased ocular surface changes demonstrated by clinical tests and impression cytology in glaucoma patients, there was no statistically and clinically significant difference in the OSDI score between glaucoma-treated patients and untreated controls. Using OSDI, Leung et $\mathrm{al}^{2}$ reported that $59 \%$ of patients had symptoms of dry eye. Although the number of preserved eye drops was positively correlated with the severity of ocular surface changes, the presence of symptoms did not correlate with the clinical tests. Another study ${ }^{21}$ found that $52 \%$ of glaucoma patients showed mild dry eye symptoms that correlated to signs only in patients with beta-blocker monotherapy. Similar to our findings, Ghosh et $\mathrm{al}^{29}$ found that the prevalence of ocular symptoms was not significantly different between treated glaucoma patients (30.7\%) versus untreated controls $(24.0 \%)$, and there was absence of significant correlation between signs and symptoms of OSD in either group. The OSDI questionnaire inquires about ocular symptoms in the previous week. However, there are natural fluctuations of symptoms during the day and patients reported increased dry eye symptoms during 
some activities, such as TV watching and driving in the evening rather than in the morning. ${ }^{13,30}$ Lack of correlation between signs and symptoms may be caused by decreased corneal sensitivity by BAK-preserved eye drops. ${ }^{16,17,31}$ In vivo confocal microscopy demonstrated a significantly lower density of subbasal corneal nerves in patients taking preserved glaucoma medications compared to both those on preservative-free medication and the control group. ${ }^{17}$ Furthermore, the OSDI questionnaire has been validated in dry eye patient population only and there is a lack of evidence for OSDI to be a valid measure of OSD in glaucoma patients. ${ }^{32}$ Recently, Mathews et $\mathrm{al}^{12}$ found that OSDI was a poor metric to evaluate OSD in glaucoma because symptoms appeared to be related mainly to visual field loss.

The limitations of our study are its cross-sectional design and the relatively small number of subjects who were examined at different times of the day and year. The majority of patients (68/79 patients) were using BAK- or BAK/ combination-preserved medications and only eleven patients were using PQ-preserved eye drops. This is a small number for a meaningful comparison to detect differences among different preservatives. However, such treatment regimen including several eye drops with different preservatives more closely resembles the clinical setting. Further prospective study including newly diagnosed treatment-naïve glaucoma patients would be more appropriate. Because of decreased corneal sensitivity in patients using preserved antiglaucoma medications, symptoms may be mild or absent despite presence of OSD. Therefore, clinicians should rely more on dry eye diagnostic tests, such as evaluation of corneal fluorescein staining and TBUT, in diagnosing OSD. The correlation of ocular surface damage to the number of preserved eye drops per day should be kept in mind when deciding about alternatives to medical treatment, as well as when using the currently more frequently prescribed glaucoma generics. Generic drugs contain the same active compound but have different excipients that may affect both tolerability and ocular surface health. For the future, we need improved clinical methods for subjective and objective assessments and for monitoring of ocular surface changes in glaucoma patients.

\section{Acknowledgment}

No financial support was received for this submission.

\section{Disclosure}

The authors report no conflicts of interest in this work.

\section{References}

1. RossiGC, TinelliC, PasinettiGM,Milano G,BianchiPE. Dryeyesyndromerelated quality of life in glaucoma patients. Eur J Ophthalmol. 2009; 19:572-579.

2. Leung EW, Medeiros FA, Weinreb RN. Prevalence of ocular surface disease in glaucoma patients. J Glaucoma. 2008;17:350-355.

3. Baudouin C, Labbe A, Liang H, Pauly A, Brignole-Baudouin F. Preservatives in eyedrops: the good, the bad and the ugly. Prog Retin Eye Res. 2010;29:312-334.

4. Lockington D, Macdonald EC, Stewart P, Young D, Caslake M, Ramaesh K. Free radicals and the $\mathrm{pH}$ of topical glaucoma medications: a lifetime of ocular chemical injury? Eye (Lond). 2012;26:734-741.

5. Baudouin C, Hamard P, Liang H, Creuzot-Garcher C, Bensoussan L, Brignole F. Conjunctival epithelial cell expression of interleukins and inflammatory markers in glaucoma patients treated over the long term. Ophthalmology. 2004;111:2186-2192.

6. Arici MK, Arici DS, Topalkara A, Guler C. Adverse effects of topical antiglaucoma drugs on the ocular surface. Clin Experiment Ophthalmol. 2000;28:113-117.

7. Herreras JM, Pastor JC, Calonge M, Asensio VM. Ocular surface alteration after long-term treatment with an antiglaucomatous drug. Ophthalmology. 1992;99:1082-1088.

8. Turacli E, Budak K, Kaur A, Mizrak B, Ekinci C. The effects of longterm topical glaucoma medication on conjunctival impression cytology. Int Ophthalmol. 1997;21:27-33.

9. Paimela T, Ryhanen T, Kauppinen A, Marttila L, Salminen A, Kaarniranta K. The preservative polyquaternium- 1 increases cytoxicity and NF-kappaB linked inflammation in human corneal epithelial cells. Mol Vis. 2012;18:1189-1196.

10. Fechtner RD, Godfrey DG, BudenzD, Stewart JA, Stewart WC, Jasek MC. Prevalence of ocular surface complaints in patients with glaucoma using topical intraocular pressure-lowering medications. Cornea. 2010;29:618-621.

11. Skalicky SE, Goldberg I, McCluskey P. Ocular surface disease and quality of life in patients with glaucoma. Am J Ophthalmol. 2012; 153(1):1-9. e2.

12. Mathews PM, Ramulu PY, Friedman DS, Utine CA, Akpek EK. Evaluation of ocular surface disease in patients with glaucoma. Ophthalmology. 2013;120:2241-2248.

13. Schiffman RM, Christianson MD, Jacobsen G, Hirsch JD, Reis BL. Reliability and validity of the ocular surface disease index. Arch Ophthalmol. 2000;118:615-621.

14. Methodologies to diagnose and monitor dry eye disease: report of the Diagnostic Methodology Subcommittee of the International Dry Eye WorkShop. Ocul Surf. 2007;5:108-152.

15. Nelson JD. Impression cytology. Cornea. 1988;7:71-81.

16. Van Went C, Alalwani H, Brasnu E, et al. Evaluation de la sensibilite corneene chez les patients traites medicalement pour un glaucome ou une hypertonie oculaire. [Corneal sensitivity in patients treated medically for glaucoma or ocular hypertension]. J Fr Ophtalmol. 2011;34:684-690. French.

17. Martone G, Frezzotti P, Tosi GM, et al. An in vivo confocal microscopy analysis of effects of topical antiglaucoma therapy with preservative on corneal innervation and morphology. Am J Ophthalmol. 2009;147: 725.e1-735.e1.

18. Baffa Ldo P, Ricardo JR, Dias AC, et al. Tear film and ocular surface alterations in chronic users of antiglaucoma medications. Arq Bras Oftalmol. 2008;71:18-21.

19. Lee SY, Wong TT, Chua J, Boo C, Soh YF, Tong L. Effect of chronic anti-glaucoma medications and trabeculectomy on tear osmolarity. Eye (Lond). 2013;27:1142-1150.

20. Nichols KK, Nichols JJ, Mitchell GL. The lack of association between signs and symptoms in patients with dry eye disease. Cornea. 2004;23:762-770.

21. Valente C, Iester M, Corsi E, Rolando M. Symptoms and signs of tear film dysfunction in glaucomatous patients. $J$ Ocul Pharmacol Ther. 2011;27:281-285. 
22. Liang H, Pauly A, Riancho L, Baudouin C, Brignole-Baudouin F. Toxicological evaluation of preservative-containing and preservative-free topical prostaglandin analogues on a three-dimensional-reconstituted corneal epithelium system. Br J Ophthalmol. 2011;95:869-875.

23. Arici MK, Arici DS, Ozec AV, Dursun A, Toker MI, Erdogan H Apoptotic effects of topical antiglaucoma medications on conjunctival epithelium in glaucoma patients. Eur J Ophthalmol. 2014;24:63-70.

24. Pisella PJ, Pouliquen P, Baudouin C. Prevalence of ocular symptoms and signs with preserved and preservative free glaucoma medication. Br J Ophthalmol. 2002;86:418-423.

25. Jaenen N, Baudouin C, Pouliquen P, Manni G, Figueiredo A, Zeyen T. Ocular symptoms and signs with preserved and preservative-free glaucoma medications. Eur J Ophthalmol. 2007;17:341-349.

26. Uusitalo H, Chen E, Pfeiffer N, et al. Switching from a preserved to a preservative-free prostaglandin preparation in topical glaucoma medication. Acta Ophthalmol. 2010;88:329-336.

27. Mastropasqua L, Agnifili L, Fasanella V, et al. Conjunctival goblet cells density and preservative-free tafluprost therapy for glaucoma: an in vivo confocal microscopy and impression cytology study. Acta Ophthalmol. 2013;91:e397-e405.
28. Sezgin Akcay BI, Guney E, Bozkurt TK, Topal CS, Akkan JC, Unlu C. Effects of polyquaternium- and benzalkonium-chloride-preserved travoprost on ocular surfaces: an impression cytology study. $J$ Ocul Pharmacol Ther. 2014;30(7):548-553.

29. Ghosh S, O’Hare F, Lamoureux E, Vajpayee RB, Crowston JG. Prevalence of signs and symptoms of ocular surface disease in individuals treated and not treated with glaucoma medication. Clin Experiment Ophthalmol. 2012;40:675-681.

30. Walker PM, Lane KJ, Ousler GW 3rd, Abelson MB. Diurnal variation of visual function and the signs and symptoms of dry eye. Cornea. 2010;29:607-612.

31. Kozobolis VP, Detorakis ET, Maskaleris G, et al. Corneal sensitivity changes following the instillation of latanoprost, bimatoprost, and travoprost eyedrops. Am J Ophthalmol. 2005;139:742-743.

32. Pflugfelder SC, Baudouin C. Challenges in the clinical measurement of ocular surface disease in glaucoma patients. Clin Ophthalmol. 2011;5:1575-1583.
Clinical Ophthalmology

\section{Publish your work in this journal}

Clinical Ophthalmology is an international, peer-reviewed journa covering all subspecialties within ophthalmology. Key topics include: Optometry; Visual science; Pharmacology and drug therapy in eye diseases; Basic Sciences; Primary and Secondary eye care; Patient Safety and Quality of Care Improvements. This journal is indexed on

Submit your manuscript here: http://www.dovepress.com/clinical-ophthalmology-journal

\section{Dovepress}

PubMed Central and CAS, and is the official journal of The Society of Clinical Ophthalmology (SCO). The manuscript management system is completely online and includes a very quick and fair peer-review system, which is all easy to use. Visit http://www.dovepress.com/ testimonials.php to read real quotes from published authors. 